Pacific Journal of 


\section{HOLOMORPHIC MAPPING OF PRODUCTS OF ANNULI IN $\boldsymbol{C}^{n}$}

\section{ERIC BEDFORD}

Let $\Omega_{1}, \Omega_{2} \subset C^{n}$ be bounded pseudoconvex Reinhardt domains with the property that $z_{1} \cdots z_{n} \neq 0$ for all $\left(z_{1}, \cdots, z_{n}\right) \in \bar{\Omega}_{j}$. A holomorphic mapping $f: \Omega_{1} \rightarrow \Omega_{2}$ is discussed in terms of the induced mapping on homology $f_{*}$ : $H_{1}\left(\Omega_{1}, \boldsymbol{R}\right) \rightarrow H_{1}\left(\Omega_{2}, \boldsymbol{R}\right)$. Specifically, there is a norm on $H_{1}\left(\Omega_{j}, R\right)$ which must decrease under $f_{*}$. As a consequence we prove that a domain $\Omega$ as above is rigid in the sense of H. Cartan: if $f: \Omega \rightarrow \Omega$ is holomorphic and $f_{*}: H_{1}(\Omega, R) \rightarrow$ $H_{1}(\Omega, R)$ is nonsingular, then $f$ is an automorphism.

1. Introduction. Let $A\left(R_{j}\right)=\left\{z \in C: 1 / R_{j}<|z|<R_{j}\right\}$ be an annulus in the complex plane. If $f: A\left(R_{1}\right) \rightarrow A\left(R_{2}\right)$ is a holomorphic mapping, then the topological behavior of $f$ is restricted in terms of the moduli $R_{1}$ and $R_{2}$ (see Schiffer [6] and Huber [4]). With the methods of Landau and Osserman [5] it will be possible to generalize this result to certain domains which are (topologically) the products of plane annuli. Domains satisfying (2) are also shown to be rigid; see Theorem 2 and Remark 1. In [1] the homology group $H_{2 n-1}$ was used to prove rigidity; here we discuss $H_{1}$.

Let $\Omega \subset C^{n}$ be a complex manifold and let

$$
\mathscr{F}=\left\{u \in C^{2}(\Omega), 0<u<1, u \text { pluriharmonic }\right\} .
$$

If $\gamma \in H_{1}(\Omega, \boldsymbol{R})$ is a homology class, then a seminorm on $\gamma$ may be defined by

$$
N\{\gamma\}=\sup _{u \in \mathscr{F}} \int_{r} d^{c} u
$$

where $d^{c}=i(\bar{\partial}-\partial)$, (see Chern, Levine, and Nirenberg [2]). If $F: \Omega_{1} \rightarrow \Omega_{2}$ is a holomorphic mapping, then the map on homology $F_{*}: H_{1}\left(\Omega_{1}, \boldsymbol{R}\right) \rightarrow H_{1}\left(\Omega_{2}, \boldsymbol{R}\right)$ must decrease this norm.

2. Computation of the intrinsic norm. We will compute this norm for domains $\Omega \subset C^{n}$ satisfying

$\Omega$ is connected, bounded, pseudoconvex, Reinhardt (i.e., (2) $\quad\left(e^{i \theta_{1}} z_{1}, \cdots, e^{i \theta_{n}} z_{n}\right) \in \Omega$ if $z \in \Omega$ and $\left.\theta_{1}, \cdots, \theta_{n} \in \boldsymbol{R}\right)$, and if $z \in \bar{\Omega}$, then $z_{1} \cdots z_{n} \neq 0$.

Let $\omega \subset \boldsymbol{R}^{n}$ be the logarithmic image of $\Omega$, i.e., 


$$
\omega=\left\{\left(\xi_{1}, \cdots, \xi_{n}\right) \in \boldsymbol{R}^{n}:\left(e^{\xi_{1}}, \cdots, e^{\xi_{n}}\right) \in \Omega\right\} .
$$

Since $\Omega$ satisfies (2), $\omega$ is convex. Choosing a point $\zeta \in \Omega$, we define $\gamma_{j} \in H_{1}(\Omega, \boldsymbol{R})$ to be the homology class of the circle $\theta \rightarrow\left(\zeta_{1}, \cdots\right.$, $\left.e^{i \theta} \zeta_{j}, \cdots, \zeta_{n}\right), 0 \leqq \theta \leqq 2 \pi$. Thus $\left\{\gamma_{1}, \cdots, \gamma_{n}\right\}$ forms a basis for $H_{1}(\Omega, \boldsymbol{R})$. For $u \in \mathscr{F}$, we set

$$
u^{0}\left(r_{1}, \cdots, r_{n}\right)=\frac{1}{(2 \pi)^{n}} \int_{0}^{2 \pi} \cdots \int_{0}^{2 \pi} u\left(r_{1} e^{i \theta_{1}}, \cdots, r_{n} e^{i \theta_{n}}\right) \cdot d \theta_{1} \cdots d \theta_{n} .
$$

Since $d^{c}$ is linear and invariant under complex rotations,

$$
\int_{\gamma_{j}} d^{c} u=\int_{\gamma_{j}} d^{c} u^{0}
$$

for all $u \in \mathscr{F}$. Let $\mathscr{F}^{0}=\left\{u \in \mathscr{F}: u=u\left(r_{1}, \cdots, r_{n}\right)\right\}$. We note that every element of $\mathscr{F}^{0}$ has the form $u=c+c_{1} \log r_{1}+\cdots+c_{n} \log r_{n}$. For $u^{0} \in \mathscr{F}^{0}$, the function $\mathfrak{I}\left(\xi_{1}, \cdots, \xi_{n}\right)=u^{0}\left(e^{\xi_{1}}, \cdots, e^{\xi_{n}}\right)$ is affine (linear plus constant). A simple computation gives

$$
\int_{r_{j}} d^{c} u^{0}=\int_{r_{j}} \frac{\partial u}{\partial r_{j}} r_{j} d \theta_{j}=2 \pi \frac{\partial \mathfrak{l}}{\partial \xi_{j}} .
$$

Thus we conclude that

$$
N\left\{a_{1} \gamma_{1}+\cdots+a_{n} \gamma_{n}\right\}=2 \pi \sup _{\mathfrak{l} \in \mathscr{L}}\left(a_{1} \frac{\partial \mathfrak{l}}{\partial \xi_{1}}+\cdots+a_{n} \frac{\partial \mathfrak{l}}{\partial \xi_{n}}\right)
$$

where

$$
\mathscr{L}(\omega)=\{\mathfrak{l}(\xi) \text { affine: } 0<\mathfrak{l}(\xi)<1, \xi \in \omega\} .
$$

We define the norm

$$
\|\mathfrak{l}\|=\max _{\bar{\omega}} \mathfrak{l}-\min _{\bar{\omega}} \mathfrak{l}
$$

so that $\mathscr{L}$ is identified via the map $\mathfrak{l} \rightarrow \mathfrak{l}-\mathfrak{l}(0)$ with $\Gamma=\{\mathfrak{l}$ linear: $\|\mathfrak{I}\| \leqq 1\}$. Clearly $\Gamma=-\Gamma$ and $\Gamma$ is convex. Let $\boldsymbol{R}_{\Gamma}^{n}$ denote the Banach space $\boldsymbol{R}^{n}$ with $\Gamma$ as its unit ball. By (3) the unit ball $B$ of $H_{1}(\Omega, \boldsymbol{R})$ is

$$
B=\left\{\gamma=\sum_{j=1}^{n} a_{j} \gamma_{j}:\left|\sum_{j=1}^{n} a_{j} \frac{\partial \mathfrak{l}}{\partial \xi_{j}}\right|<\frac{1}{2 \pi} \text { for } \mathfrak{l} ;\|\mathfrak{l}\|<1\right\}
$$

which is $1 / 2 \pi$ times the unit ball of $\left(\boldsymbol{R}_{\Gamma}^{n}\right)^{\prime}$.

If $\omega=-\omega$, then $\left(\boldsymbol{R}_{\omega}^{n}\right)^{\prime}=\boldsymbol{R}_{2 \Gamma}^{n}$, and thus $B$ is naturally identified in $R_{\omega}^{n}$ as $B=(1 / \pi) \omega$. If $\omega$ is any convex set, then the convex set $\tilde{\omega}=\pi B \subset \boldsymbol{R}^{n}$ satisfies $\tilde{\omega}=-\tilde{\omega}$ and has the same unit ball, $B$, as $\omega$. For a general convex set $\omega$, we may assume that $0 \in \omega$ and let $\rho(\xi)$ be its support function, i.e., $\rho(\xi)$ is the distance from 0 of the hyper- 
plane which supports $\omega$ and has outward normal $\xi$. It follows that

$$
\Gamma=\left\{\mathfrak{l}(\xi)=\sum c_{j} \xi_{j}:\left(\sum c_{j}^{2}\right)^{1 / 2} \leqq \frac{1}{(\rho(c)+\rho(-c))}\right\} .
$$

In terms of the basis $\left\{d \theta_{1}, \cdots, d \theta_{n}\right\}, \Gamma$ may be identified as a subset of $H^{1}(\Omega, \boldsymbol{R})$, and so $H^{1}$ inherits the dual norm. Thus, for each $a \epsilon$ $H^{1}(\Omega, \boldsymbol{R})$ with $a \in \partial \Gamma$, there exists $\gamma \in H_{1}(\Omega, \boldsymbol{R})$ such that $\gamma \cdot a=N\{\gamma\}$.

For $u \in \mathscr{F}, \mathfrak{l} \in \Gamma$, we will use the notation:

$$
\begin{aligned}
L u(\xi) & =u^{0}\left(e^{\xi}\right) \\
\tilde{I}(z) & =\mathfrak{I}(\log |z|) .
\end{aligned}
$$

It is useful to know, given a homology class $\gamma \in H_{1}(\Omega, Z)$, whether there is an imbedded annulus $\varphi: A(R) \rightarrow \Omega$ such that $\varphi_{*}(|z|=1)=\gamma$ and $N\{|z|=1\}=N\{\gamma\}$. We do not know this in general, but this happens when $\omega=-\omega$. For integers $m_{1}, \cdots, m_{n}$, we define the map $\varphi: A(R) \rightarrow C^{n}$ by $\varphi(\tau)=\left(\tau^{m_{1}}, \cdots, \tau^{m_{n}}\right)$, and thus $\varphi_{*}(|z|=1)=\sum m_{j} \gamma_{j}$. It is easily seen that $\varphi(A(R)) \subset \Omega$ for $\log R=\mu$ if $\left(\mu m_{1}, \cdots, \mu m_{n}\right) \in \omega$. By the identification $B=(1 / \pi) \omega$, we have

$$
N(\sigma)=\frac{\pi}{\mu}=N\left\{\varphi_{*}(\sigma)\right\}=N\left\{\sum m_{j} \gamma_{j}\right\}
$$

for $\mu=\log R$ and $\mu\left(m_{1}, \cdots, m_{n}\right) \in \partial \omega$.

3. Extremal functions. To study holomorphic mappings we will need to know that the function achieving the supremum in (1) is unique.

Proposition 1. If $\gamma$ is the homology class of $\{|z|=1\}$ in the annulus $A(R)$, then

$$
u=\frac{\log R|z|}{2 \log R}
$$

is the unique function in $\mathscr{F}$ satisfying

$$
N\{\gamma\}=\int_{\gamma} d^{c} u
$$

If $v \in \mathscr{F}$ satisfies

$$
c N\{\gamma\}=\int_{r} d^{c} v
$$

then

$$
\frac{1}{2 \pi} \int_{0}^{2 \pi}\left|v\left(r e^{i \theta}\right)-u(r)\right| d \theta \leqq 4(1-c)
$$


for $1 / R<r<R$.

Proof. The first assertion is well known. The idea of the proof is that if $v \in \mathscr{F}$, and if $\{u>v\}$ is nonempty, then the homology class of $\gamma^{\prime}=\partial\{u>v\}$ is homologous to $\gamma$. Thus if $v$ satisfies (5), then

$$
\int_{r} d^{c}(u-v)=\int_{r^{\prime}} d^{c}(u-v)=0 .
$$

Thus $\nabla(u-v)=0$ on $\gamma^{\prime}$, and by unique continuation, $u=v$ on $A(R)$. For details, see Landau and Osserman [5], or [1].

For the second assertion, we consider the Laurent expansion

$$
v(z)=c u(z)+c_{0}+\operatorname{Re} g(z)
$$

where $g(z)=\sum_{j \neq 0} c_{j} z^{j}$. Since $\operatorname{Re} g(z)$ is a bounded harmonic function on $A(R)$, it has nontangential boundary limits a.e. on $|z|=R$ and $|z|=1 / R$. It follows that

$$
\int_{0}^{2 \pi} \operatorname{Re} g\left(r e^{i \theta}\right) d \theta=0
$$

for $1 / R \leqq r \leqq R$. Since $v \in \mathscr{F}$, it follows that $c_{0}+c \leqq 1$ and $\operatorname{Re} g(z) \leqq 1-c-c_{0}$ for $|z|=R$; and $c_{0} \geqq 0, \operatorname{Re} g(z) \geqq-c_{0}$ for $|z|=$ $1 / R$. Therefore

$$
\frac{1}{2 \pi} \int_{0}^{2 \pi}\left|\operatorname{Re} g\left(r e^{i \theta}\right)\right| d \theta \leqq 2(1-c)
$$

for $r=R$ and $r=1 / R$. Since Re $g$ is harmonic on $A(R)$, this bound holds for $1 / R \leqq r \leqq R$. Thus

$$
\frac{1}{2 \pi} \int_{0}^{2 \pi}\left|u(r)-v\left(r e^{i \theta}\right)\right| d \theta \leqq 1-c+c_{0}+\frac{1}{2 \pi} \int_{0}^{2 \pi}\left|\operatorname{Re} g\left(e^{i \theta}\right)\right| d \theta
$$

which gives the desired estimate.

Proposition 2. Let $\Omega$ satisfy (2), and let $\gamma \in H_{1}(\Omega, \boldsymbol{R})$ be given. If $u$ satisfies (5), then $u(z)=u^{0}(z)$ for all $z \in \Omega$ such that $\log |z|$ belongs to the convex hull of $\{\xi \in \partial \omega: L u(\xi)=0$ or 1$\}$. In particular, if

there exist

$p_{0} p_{1} \in \bar{\omega}, L u\left(p_{1}\right)=1, L u\left(p_{0}\right)=0$

$c=\left(c_{1}, \cdots, c_{n}\right)=p_{1}-p_{0}$ and the

set $\left\{c_{1}, \cdots, c_{n}\right\}$ is rationally

independent 
then $u(z)=u^{0}(z)$ for all $z \in \Omega$.

Proof. Let us begin by recalling that $\int_{r} d^{c}\left(u^{0}-u\right)=0$ for all $\gamma \in H_{1}(\Omega, \boldsymbol{R})$. Thus there is a holomorphic function $f \in \mathcal{O}(\Omega)$ such that $u=u^{0}+\operatorname{Re} f$. If the first part of the proposition is proved, then it follows that $\operatorname{Re} f(z)=0$ on $S=\{z \in \Omega: \log |z|=\lambda c, \lambda \in R\}$, if $p_{0}=0$. If (6) holds there is a one-dimensional complex manifold $M=$ $\left\{\left(\tau^{c_{1}}, \cdots, \tau^{c_{n}}\right): \tau \in C\right\} \cap \Omega$ which is dense in $S$. Since $M$ is complex, it follows that $f=0$ on $M$. Thus $f=0$ on $S$, and so $f=0$ on $\Omega$.

Now we establish the first part of the proposition. Let $p_{0}, p_{1} \in$ $\partial \omega$ be such that $L u\left(p_{0}\right)=0$ and $L u\left(p_{1}\right)=1$. Without loss of generality we may assume that $p_{1}=-p_{0}$. We first consider the case where the ratios $c_{j} / c_{k}$ are all rational. Thus there are integers $\left(m_{1}, \cdots, m_{n}\right)$ such that $c_{j}=\mu m_{j}$ for some $\mu \in \boldsymbol{R}$. The mapping $\varphi_{m}(\tau)=\left(\tau^{m_{1}}, \cdots, \tau^{m_{n}}\right)$ maps the annulus $A\left(e^{\mu}\right)$ into $\Omega$, and the logarithmic image of $\varphi\left(A\left(e^{\mu}\right)\right)$ is the segment $\left(p_{0}, p_{1}\right)$. It follows that $u(\varphi)$ and $u^{0}(\varphi)$ both satisfy (5), and thus by Proposition $1 u(\varphi)=u^{0}(\varphi)$ on $A$. Since this argument applies to all mappings $\varphi(\tau)=\left(e^{i \theta_{1}} \tau^{m_{1}}, \cdots, e^{i \theta_{n}} \tau^{m_{n}}\right)$, we conclude that $u(z)=u^{0}(z)$ for all $z$ such that $\log |z| \in\left(p_{0}, p_{1}\right)$.

For general $c$, we may take a sequence $\left\{c^{s}\right\}, c^{s}=\mu_{s}\left(m_{1}^{s}, \cdots, m_{n}^{s}\right)$, $\mu_{s} \in \boldsymbol{R}, m_{i}^{s} \in \boldsymbol{Z}$ such that $\pm c^{s} \in \bar{\omega}$ and $c^{s}$ converges to $p_{1}$. As before we set $\varphi_{m^{s}}=\varphi_{s}: A\left(e^{\mu_{s}}\right) \rightarrow \Omega$. Thus

$$
u^{0}\left(\varphi_{s}(z)\right)=\frac{\log e^{\mu_{s}}|z|}{2 \log e^{\mu_{s}}}+\varepsilon(s)
$$

where $\varepsilon(s)$ is a function on $A\left(e^{\mu_{s}}\right)$ such that

$$
\lim _{s \rightarrow \infty}\|\varepsilon(s)\|=0\left(\text { here }\|\varepsilon(s)\|=\sup _{A\left(e^{\mu_{s}}\right)}|\varepsilon(s)|\right) .
$$

If $\sigma$ is the class of $\{|z|=1\}$ in $A\left(e^{\mu_{s}}\right)$ then

$$
\int_{\sigma} d^{c} u^{0}\left(\varphi_{s}\right) \geqq(1-\|\varepsilon(s)\|) N\{\sigma\} .
$$

Since

$$
\int_{\left(\varphi_{s}\right) * \sigma} d^{c} u=\int_{\left(\varphi_{s}\right) * \sigma} d^{c} u^{0}
$$

we have

$$
\int_{\sigma} d^{c} u\left(\varphi_{s}\right) \geqq(1-\|\varepsilon(s)\|) N\{\sigma\} .
$$

By Proposition 1, then, 


$$
\frac{1}{2 \pi} \int_{0}^{2 \pi}\left|u\left(\varphi_{s}\left(r e^{i \theta}\right)\right)-u^{0}\left(\varphi_{s}(r)\right)\right| d \theta \leqq 4\|\varepsilon(s)\|
$$

Clearly the same holds if $\varphi_{s}$ is replaced by $\varphi(\gamma)=\left(e^{i \theta_{1}} \tau^{m_{1}}, \cdots, e^{i \theta_{n}} \tau^{m} n\right)$ with $\theta_{1}, \cdots, \theta_{n} \in \boldsymbol{R}$.

Finally we will show that $u(r)=u^{0}(r)$ for $r=\lambda c, 0<\lambda<1$. If this does not hold, then there exists $\delta>0$ such that $\left|u(z)-u^{0}(|z|)\right|>\delta$ for all $z$ such that $|z-r|<\delta$. Now we may cover the set $T=$ $\left\{z \in \Omega:\left|z_{j}\right|=r_{j}\right\}$ with $K$ balls ( $K$ large) of radius $\delta$ and centers $q_{1}, \cdots, q_{K} \in T$. At least one of these balls has the property that

$$
\frac{2 \pi}{K} \leqq \text { measure }\left\{0<\theta<2 \pi:\left|\varphi_{s}\left(\rho e^{i \theta}\right)-q_{j}\right|<\delta\right\},
$$

where $\varphi_{s}(\rho)=r$. Denote $\operatorname{Arg}\left(q_{j}\right)$ by $\left(\psi_{1}, \cdots, \psi_{n}\right)$. It follows that

$$
\begin{aligned}
& \int_{0}^{2 \pi}\left|u\left(\widetilde{\varphi}_{s}\left(\rho e^{i \theta}\right)\right)-u^{0}(\boldsymbol{r})\right| d \theta \\
& \quad \geqq \delta \text { measure }\left\{0<\theta<2 \pi:\left|\widetilde{\varphi}_{s}\left(\rho e^{i \theta}\right)-r\right|<\delta\right\} \geqq \frac{2 \pi \delta}{K}
\end{aligned}
$$

where $\widetilde{\varphi}_{s}=\left(e^{-i \psi_{1}} \tau^{m_{1}}, \cdots, e^{-i \psi_{n}} \tau^{m_{n}}\right)$. Since this contradicts our previous estimate, we conclude that $u(z)=u^{0}(\boldsymbol{z})$ if $|\boldsymbol{z}|=r$, which was what we wanted to prove.

Proposition 3. Let $\omega \subset \boldsymbol{R}^{n}$ be a bounded convex set. Given $c \in \boldsymbol{R}^{n}, c \neq 0$, there exists $u \in \mathscr{F}, p_{0}, p_{1} \in \partial \omega$ such that $p_{1}-p_{0}=\lambda c$, $\lambda \in \boldsymbol{R}$, and $L u\left(p_{j}\right)=j$ for $j=0,1$. Furthermore, there exist $u_{1}, \cdots, u_{n} \in \mathscr{F}$ satisfying (6) and such that $L u_{1}, \cdots$, Lu $u_{n}$ are linearly independent.

Proof. Let us first suppose that $\partial \omega$ is smooth and strictly convex. Let $\alpha: S^{n-1} \rightarrow \partial \omega$ be the Gauss map, i.e., the outward normal to $\partial \omega$ at $\alpha(\xi)$ is $\xi$. Consider the map $\beta: S^{n-1} \rightarrow S^{n-1}$ given by

$$
\beta(\xi)=\frac{\alpha(\xi)-\alpha(-\xi)}{|\alpha(\xi)-\alpha(-\xi)|} .
$$

Clearly $\beta(\xi) \cdot \xi>0$, and thus $\beta$ has degree 1 , so that $\beta$ is onto. Let $\xi_{0}$ be a vector such that $\beta\left(\xi_{0}\right)=c /|c|$. Then we take $p_{1}=\alpha\left(\xi_{0}\right), p_{0}=$ $\alpha\left(-\xi_{0}\right)$, and grad $L u=\beta(\xi)$.

For general $\omega$, we take an increasing sequence $\left\{\omega_{j}\right\}$ of smoothly bounded strictly convex sets. If $u^{j}, p_{0}^{j}, p_{1}^{j}$ have the desired properties on $\omega_{j}$, we pass to a convergent subsequence to obtain $u, p_{0}, p_{1}$.

Now we show that we can obtain the family $\left\{u_{1}, \cdots, u_{n}\right\}$. Let us suppose that we have found $\left\{u_{1}, \cdots, u_{j}\right\}$ with $\left\{L u_{1}, \cdots, L u_{j}\right\}$, 
$1 \leqq j<n$, linearly independent and satisfying (6). Pick $c \in$ $\bigcap_{k \leqq j} \operatorname{Ker} L u_{k}, c \neq 0$. It follows that if $u_{j+1}$ satisfies the conclusion of the first part of the proposition, then $\left\{L u_{1}, \cdots, L u_{j+1}\right\}$ are linearly independent. Now we perturb $c$ slightly so that (6) is satisfied and the set is still independent.

4. Application to holomorphic mappings. Let $F: \Omega_{1} \rightarrow \Omega_{2}$ be a holomorphic mapping of domains satisfying (2). Then by the integer matrix $T_{F}$ we will denote the map on integral homology classes $F_{*}=T_{F}: Z^{n} \rightarrow Z^{n}$ in terms of basis $\left\{\gamma_{1}, \cdots, \gamma_{n}\right\}$. It follows that $T_{F}\left(B_{1}\right) \subset B_{2}$ and $T_{F}^{\prime}\left(\Gamma_{2}\right) \subset \Gamma_{1}$, where $T_{F}^{\prime}$ is the transpose of $T_{F}$, and $T_{F}^{\prime}$ gives the action of $F^{*}$ on $H^{1}$. If $\mathfrak{l}(\xi)=\sum c_{j} \xi_{j}$, then $F^{*} d^{\mathcal{\imath} \mathfrak{Y}}$ represents the same cohomology class as $T_{F}^{\prime}(c)$. Writing $u(z)=\tilde{I}(F(z))$ we have $L u(\xi)=T_{F}^{\prime}(c) \cdot \xi$.

THEOREM 1. Let $\Omega_{1}, \Omega_{2}$ satisfy (2), and assume that $\omega_{1}=-\omega_{1}$, $\omega_{2}=-\omega_{2}$. Let $T$ be an $n \times n$ matrix with integer entries. There exists a holomorphic mapping $F: \Omega_{1} \rightarrow \Omega_{2}$ with $T_{F}=T$ if and only if $T\left(\omega_{1}\right) \subset \omega_{2}$. Furthermore $T\left(\omega_{1}\right)=\omega_{2}$ (i.e., $F_{*}$ is an isometry) if and only if $F$ is a proper covering map, and in this case $F$ has the form

$$
F(\boldsymbol{z})=\left(e^{i \theta_{1}} z^{t_{1}}, e^{i \theta_{n}} z^{t_{n}}\right)
$$

where $\theta_{1}, \cdots, \theta_{n} \in R$ and $t_{1}, \cdots, t_{n}$ are the rows of $T$.

Proof. Let $F: \Omega_{1} \rightarrow \Omega_{2}$ be given. Since $F_{*}$ must be normdecreasing, and since $1 / \pi \omega_{j}=B_{j}$, it follows that $T\left(\omega_{1}\right) \subset \omega_{2}$. Conversely, if $T\left(\omega_{1}\right) \subset \omega_{2}$, we set $F\left(z_{1}, \cdots, z_{n}\right)=\left(z^{t_{1}}, \cdots, z^{t_{n}}\right)$. Exponentiating the inclusion $T\left(\omega_{1}\right) \subset \omega_{2}$, we obtain $F\left(\Omega_{1}\right) \subset \Omega_{2}$.

Now we assume that $T_{F}$ is an isometry, and let $\left\{u_{1}, \cdots, u_{n}\right\} \subset$ $\mathscr{F}^{0}\left(\Omega_{1}\right)$ be the set constructed in Proposition 3 . We may assume that $d^{c} u_{j} \in \partial \Gamma$, so there exists $\left\{\gamma_{1}, \cdots, \gamma_{n}\right\} \subset H_{1}\left(\Omega_{1}, \boldsymbol{R}\right)$ such that $N\left\{\gamma_{j}\right\}=\int_{\gamma_{j}} d^{c} u_{j}$. Now we pick $u_{1}^{\prime}, \cdots, u_{n}^{\prime} \in \mathscr{F}^{0}\left(\Omega_{2}\right)$ such that the cohomology class of $d^{c} u_{j}$ is the same as $F^{*}\left(d^{c} u_{j}^{\prime}\right)$. Thus

$$
\int_{r_{j}} d^{c} u_{j}=N\left\{\gamma_{j}\right\}=N\left\{F_{*} \gamma_{j}\right\}=\int_{\gamma_{j}} F^{*}\left(d^{c} u_{j}\right) \text {. }
$$

Since $F$ is holomorphic,

$$
\int_{r_{j}} F^{*}\left(d^{c} u_{j}\right)=\int_{r_{j}} d^{c}\left(u_{j}^{\prime}(F)\right) .
$$

Since $u_{j}$ satisfies (6), we conclude by Proposition 2, that $u_{j}=$ $u_{j}^{\prime}(F)$. This gives $n$ independent equations which have the form 


$$
\sum_{i=1}^{n} c_{i j} \log \left|z_{i}\right|=\sum_{i=1}^{n} c_{i j}^{\prime} \log \left|F_{i}(z)\right|
$$

for $j=1, \cdots, n$. Thus $\log \left|F_{i}(z)\right|=\sum a_{i j} \log \left|z_{j}\right|, i=1, \cdots, n$. Since $T_{F}=T$, it follows that $a_{i j}=t_{i j}$, and so $F$ has the desired form. Thus

$$
\frac{\partial F_{i}}{\partial z_{j}}=\frac{t_{i j}}{z_{j}} F_{i}
$$

so that $\operatorname{det}\left(\partial F_{i} / \partial z_{j}\right)=\left(\prod_{k=1}^{n} F_{k} / z_{k}\right) \operatorname{det} T \neq 0$. Since $T\left(\omega_{1}\right)=\omega_{2}$ it follows that $F$ is in fact a covering map and is proper.

Conversely, we shows that if $F$ is a covering, then $F_{*}$ is an isometry. We consider first the one-dimensional case $f: A\left(R_{1}\right) \rightarrow A\left(R_{2}\right)$, where $f$ is a d-to-1 covering. If $\varphi: A\left(R_{2}^{1 / d}\right) \rightarrow A\left(R_{2}\right)$ is given by $\varphi(z)=z^{d}$, then taking a suitable branch of $\phi^{-1}(f)$ we obtain a biholomorphism between $A\left(R_{1}\right)$ and $A\left(R_{2}^{1 / d}\right)$. Since $R_{1}=R_{2}^{1 / d}, f_{*}$ is an isometry.

For the general case, we consider integral homology classes $\gamma^{\prime}=\sum m_{j} \gamma_{j}^{\prime} \in H_{1}\left(\Omega_{2}, Z\right)$. Let $\varphi: A^{\prime} \rightarrow \Omega_{2}$ be an imbedding of an annulus so that $\varphi_{*}(\sigma)=\gamma^{\prime}$ and (4) holds. If we set $A=F^{-1}\left(\varphi A^{\prime}\right)$, then $F_{\mid A}: A \rightarrow \varphi A^{\prime}$ is a covering. $F$ is proper, so $F^{-1} \gamma^{\prime}$ is a closed curve in $\Omega_{1}$; thus $A$ is a 1 -dimensional annulus and so $\left(F_{\mid A}\right)_{*}$ is an isometry. We let $\sigma$ be the generator of $H_{1}(A, Z)$, and we let $\gamma=\gamma_{\sigma}$ be the induced element of $H_{1}\left(\Omega_{1}, Z\right)$. Thus $F_{*}(\gamma)=\gamma^{\prime}$, and so $N\{\gamma\} \geqq$ $N\left\{\gamma^{\prime}\right\}$. On the other hand, since $A \subset \Omega_{1}$,

$$
N\left\{\gamma^{\prime}\right\}=N\left\{\sigma^{\prime}\right\}=N\{\sigma\} \geqq N\{\gamma\},
$$

and so $N\{\gamma\}=N\left\{F_{*}(\gamma)\right\}$. Since this holds for all integral classes in $H_{1}\left(\Omega_{2}, \boldsymbol{R}\right)$, it follows that $F_{*}$ is an isometry.

TheOREM 2. Let $\Omega_{1}, \Omega_{2}$ satisfy (2). If $F: \Omega_{1} \rightarrow \Omega_{2}$ is a holomorphic mapping such that $F_{*}: H_{1}\left(\Omega_{1}, \boldsymbol{R}\right) \rightarrow H_{1}\left(\Omega_{2}, \boldsymbol{R}\right)$ is an isometry, then $F$ is a covering map of the form

$$
F(z)=\left(c_{1} z^{t_{1}}, \cdots, c_{n} z^{t_{n}}\right)
$$

where $c_{1}, \cdots, c_{n} \in C$ and $t_{1}, \cdots, t_{n}$ are the rows of $T_{F}$. In particular, if $\Omega_{1}=\Omega_{2}$ and $F_{*}$ is nonsingular, then $F$ is a biholomorphism.

Proof. We repeat the appropriate portion of the proof of Theorem 1 and conclude that if $F_{*}$ is an isometry, then

$$
c_{0 j}+\sum_{i=1}^{n} c_{i j} \log \left|z_{i}\right|=c_{0 j}^{\prime}+\sum_{i=1}^{n} c_{i j}^{\prime} \log \left|F_{i}(z)\right|
$$

for $j=1, \cdots, n$. Thus $\left|F_{j}(z)\right|=b_{j}\left|z_{1}\right|^{b_{1 j}} \cdots\left|z_{n}\right|^{b_{n j}}$, and so $F$ has the desired form since $F_{*}=T_{F}$. As before, $\operatorname{det}\left(\partial F_{i} / \partial z_{j}\right) \neq 0$. To show that $F$ is a covering, we show that $F$ is proper. We have already 
shown that $F(z)=\left(c_{1} z^{t_{1}}, \cdots, c_{n} z^{t_{n}}\right)$ and so for $\mathfrak{l}^{\prime} \in \Gamma_{2}, L \tilde{\mathfrak{l}}^{\prime}(F) \in \Gamma_{1}$. We set $U_{j}(z)=\sup _{t \in \partial \Gamma j} \tilde{\mathfrak{l}}(z)$. By the convexity of $\omega_{j}, U_{j}$ is an exhaustion for $\Omega_{j}: \partial \Omega_{j}=\left\{z \in \bar{\Omega}_{j}: U_{j}(z)=1\right\}$. As was noted above,

$$
T_{F}^{\prime} \mathfrak{l}^{\prime}=\tilde{\mathfrak{l}}^{\prime}(\log |F|)
$$

for $\mathfrak{I}^{\prime} \in \Gamma_{2}$. Since $F_{*}$ is an isometry, $F^{*} \Gamma_{2}=\Gamma_{1}$, and so

$$
U_{1}(z)=U_{2}(F(z)) \text {. }
$$

Thus $F$ is proper.

In case $\Omega_{1}=\Omega_{2}$, then $F_{*} B_{1} \subset B_{1}$. Since $T_{F}$ has integer coefficients and is invertible, $\operatorname{det} T_{F}= \pm 1$. Thus $T_{F}$ preserves volume, and so $T_{F} B_{1}=B_{1}$. The inverse mapping is easily constructed as $G(z)=$ $\left(\zeta^{s_{1}}, \cdots, \zeta^{s_{n}}\right)$ where $\zeta_{j}=z_{j} / c_{j}$ and $s_{j}$ is the $j$ th row of the inverse $S=T^{-1}$.

REMARK 1. It follows that domains satisfying (2) are rigid in the sense of H. Cartan [2]: if $f: \Omega \rightarrow \Omega$ is holomorphic and induces a nonsingular mapping on $H_{1}(\Omega, \boldsymbol{R})$, then $f$ is an automorphism. By topological considerations, it follows that if $f_{*}$ is nonzero on the generator of $H_{n}(\Omega, \boldsymbol{R})$, then $f_{*}$ is nonsingular on $H_{1}(\Omega, \boldsymbol{R})$ and is thus an automorphism. If $T$ is a complex 1-dimensional torus and if $D \subset C$ is a disk, then $T \times D$ is a complex manifold homeomorphic to $A(R) \times A(R)$ but is not rigid. We would expect, however, that a bounded domain in $C^{n}$, homeomorphic to $A(R) \times \cdots \times A(R)$, would be rigid.

REMARK 2. The problem of finding nontrivial automorphisms (i.e., other than $\left.z \rightarrow\left(e^{i \theta_{1}} z_{1}, \cdots, e^{i \theta_{n}} z_{n}\right)\right)$ of domain satisfying (2) is thus reduced to finding $T \in G L(n, Z)$ such that $T B=B$. For instance, if $1 \leqq p<\infty$, this argument shows that the automorphisms of the domain

$$
\Omega=\left\{z \in C^{n}: \sum_{j=1}^{n}\left(\log \frac{\left|z_{j}\right|}{R_{j}}\right)^{p}<1\right\}
$$

are generated by the nontrivial automorphisms $z \rightarrow\left(z_{1}, \cdots, z_{j}^{-1}, \cdots, z_{n}\right)$ and $z_{j} \rightarrow z_{k}$ if $R_{j}=R_{k}$. Since a "generic" norm on $\boldsymbol{R}^{n}$ does not have any nontrivial isometries, a "generic" domain satisfying (2) has only trivial automorphisms.

REMARK 3. Let us consider domains satisfying (7) for some fixed $j$ :

$\Omega$ is connected, bounded, pseudoconvex, Reinhardt, if $z \in \bar{\Omega}$, (7) then $z_{1}, \cdots, z_{j} \neq 0$, and there are points $P_{j+1}, \cdots, P_{n} \in \bar{\Omega}$ such that the $k$ th coordinate of $P_{k}$ is 0 . 
Let $p: \boldsymbol{C}^{n} \rightarrow \boldsymbol{C}^{j}$ be projection onto the first $j$ variables, and set $\Omega_{0}=$ $p(\Omega)$. Looking at the logarithmic image of $\Omega$, which is convex, one may deduce that $\Omega_{0} \times\{0\} \subseteq \Omega$. By the norm-decreasing property of inclusion $i: \Omega_{0} \rightarrow \Omega$ and projection $p: \Omega \rightarrow \Omega_{0}$, it follows that $i_{*}$ and $p_{*}$ are isometries of $H_{1}$. Thus the norm of a domain satisfying (7) may be computed in terms of $\Omega_{0}$, which satisfies (2).

REMARK 4. The following observation extends Proposition 2.

Proposition 4. Let $\Omega$ satisfy (2), and assume that for each $p \in \partial \omega$ there is a unique supporting hyperplane at $p$. Then for each homology class $\gamma \in H_{1}(\Omega, \boldsymbol{R})$ there is a unique function $u \in \mathscr{F}^{0}$ such that $N\{\gamma\}=\int_{r} d^{c} u$.

Proof. We show that the $\mathfrak{l} \in \mathscr{L}$ which achieves the supremum in (3) is unique. Suppose, to the contrary, that $\mathfrak{l}_{1}, \mathfrak{l}_{2} \in \mathscr{L}$ have this property. Then so does $\mathfrak{l}=\left(\mathfrak{l}_{1}+\mathfrak{l}_{2}\right) / 2$. Since $\mathfrak{l}$ is extremal, there must be points $p^{\prime}, p^{\prime \prime} \in \partial \omega$ such that $\mathfrak{I}\left(p^{\prime}\right)=0$ and $\mathfrak{l}\left(p^{\prime \prime}\right)=1$. Thus we must have $\mathfrak{I}_{1}\left(p^{\prime \prime}\right)=\mathfrak{l}_{2}\left(p^{\prime \prime}\right)=1$, and so the half spaces $\left\{\xi: \mathfrak{l}_{1}(\xi) \leqq 1\right\}$ and $\left\{\xi: \mathfrak{l}_{1}(\xi) \leqq 1\right\}$ both support $\omega$ at $p^{\prime \prime}$. By assumption, then, $\mathfrak{l}_{1}$ is a multiple of $\mathfrak{l}_{2}$. Since $\mathfrak{l}_{1}\left(p^{\prime}\right)=\mathfrak{l}_{2}\left(p^{\prime \prime}\right)=0$, it follows that $\mathfrak{I}_{1}=\mathfrak{l}_{2}$, which completes the proof.

EXAMPLE. If $\Omega=A(R) \times A(R)$, then the homology class $\gamma=$ $\gamma_{1}+\gamma_{2}$ has norm $\pi / \log R$. For $0 \leqq \lambda \leqq 1$, the function

$$
u_{\lambda}=\frac{1}{\log R}\left(\lambda \log \left|z_{1}\right|+(1-\lambda) \log \left|z_{2}\right|\right)
$$

belongs to $\mathscr{F}^{0}$ and satisfies (5), and so the extremal function is not unique.

A slight modification of the proof of Proposition 4 shows that uniqueness holds if $\gamma=\sum a_{j} \gamma_{j}$ does not have the property:

if $t_{0}>0$ is such that $t_{0} a \in \partial \Gamma$, then there is a segment $I \subset \partial \Gamma$ containing $t_{0} a$ with $I \perp a$.

Clearly there is a dense subset of $H_{1}$ where (8) does not hold.

\section{REFERENCES}

1. E. Bedford and D. Burns, Holomorphic mapping of annuli in $\mathbf{C}^{n}$ and the associated extremal function, Ann. Scuola Norm. Sup. Pisa, 3 (1979), 381-414.

2. H. Cartan, Sur les fonctions de plusieurs variables complexes, Math. Z., 35 (1932), 760-773. 
3. S.-S. Chern, H. Levine, and L. Nirenberg, Intrinsic norms on a complex manifold, Global Analysis. Papers in Honor of K. Kodaira. Princeton Univ. Press, 1969, 119-139. 4. H. Huber, Ueber analytische Abbildungen von Ringgebieten in Ringgebiete, Compos. Math., 9 (1951), 161-168.

5. H. Landau and R. Osserman, On analytic mappings of Riemann surfaces, J. Analyse Math., 7 (1959-60), 249-279.

6. M. Schiffer, On the modules of doubly connected domains, Quart. J. Math., 17 (1946), 197-213.

Received April 24, 1978.

PRINCETON UNIVERSITY

PRINCETON, NJ 08544 

.././. ./FrontMatter/paper .pdf 


\section{Pacific Journal of Mathematics \\ Vol. 87, No. $2 \quad$ February, 1980}

Theagenis Abatzoglou, Unique best approximation from a $C^{2}$-manifold in Hilbert space ................................. 233

Gerald Arthur Anderson, $\Lambda$-homology cobordism bundles............. 245

Eric Bedford, Holomorphic mapping of products of annuli in $\mathbf{C}^{n} \ldots \ldots \ldots 271$

Gunnar Carlsson, On the stable splitting of $b o \wedge b o$ and torsion operations in connective $K$-theory .......................... 283

Lester Eli Dubins and David Samuel McIntyre Margolies, Naturally integrable functions ................................. 299

Leo Egghe, The Radon-Nikodým property, $\sigma$-dentability and martingales in locally convex spaces ............................. 313

Irving Leonard Glicksberg, Maps preserving translates of a function ..... 323

Hugh M. Hilden and Robert D. Little, Cobordism of branched covering

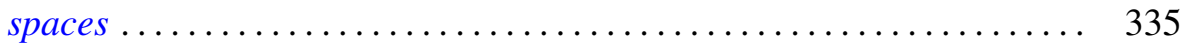

Russell Allan Johnson, Almost-periodic functions with unbounded integral .......................................... 347

Bruce Stephen Lund, The endomorphisms of a Dirichlet algebra ........ 363

John Henry McCleary, Mod $p$ decompositions of H-spaces; another approach........................................ 373

Arlan Bruce Ramsay, Subobjects of virtual groups ................ 389

Thomas R. Savage, Generalized inverses in regular rings ............ 455 Jaak Vilms, On curvature operators of bounded rank ......... 